\title{
Origin of the Difference in the Resistivity of As-Grown Focused-Ion- and Focused-Electron-Beam-Induced Pt Nanodeposits
}

\author{
J. M. De Teresa, ${ }^{1,2}$ R. Córdoba, ${ }^{2,3}$ A. Fernández-Pacheco, ${ }^{1,2,3}$ O. Montero, ${ }^{2,3}$ \\ P. Strichovanec, ${ }^{3}$ and M. R. Ibarra ${ }^{1,2,3}$ \\ ${ }^{1}$ Instituto de Ciencia de Materiales de Aragón, Facultad de Ciencias, Consejo Superior de Investigaciones Científicas (CSIC), \\ Universidad de Zaragoza, 50009 Zaragoza, Spain \\ ${ }^{2}$ Departamento de Fìsica de la Materia Condensada, Facultad de Ciencias, Universidad de Zaragoza, \\ 50009 Zaragoza, Spain \\ ${ }^{3}$ Instituto de Nanociencia de Aragón, Universidad de Zaragoza, 50009 Zaragoza, Spain \\ Correspondence should be addressed to J. M. De Teresa, deteresa@unizar.es
}

Received 20 October 2008; Revised 19 January 2009; Accepted 3 February 2009

Recommended by Xuedong Bai

\begin{abstract}
We study the origin of the strong difference in the resistivity of focused-electron- and focused-Ga-ion-beam-induced deposition (FEBID and FIBID, resp.) of Pt performed in a dual beam equipment using $\left(\mathrm{CH}_{3}\right)_{3} \mathrm{Pt}\left(\mathrm{CpCH}_{3}\right)$ as the precursor gas. We have performed in-situ and ex-situ resistance measurements in both types of nanodeposits, finding that the resistivity of Pt by FEBID is typically four orders of magnitude higher than Pt by FIBID. In the case of Pt by FEBID, the current-versus-voltage dependence is nonlinear and the resistance-versus-temperature behavior is strongly semiconducting, whereas Pt by FIBID shows linear currentversus-voltage dependence and only slight temperature dependence. The microstructure, as investigated by high-resolution transmission electron microscopy, consists in all cases of Pt single crystals with size about $3 \mathrm{~nm}$ embedded in an amorphous carbonaceous matrix. Due to the semiconducting character of the carbon matrix, which is the main component of the deposit, we propose that the transport results can be mapped onto those obtained in semiconducting materials with different degrees of doping. The different transport properties of Pt by FEBID and FIBID are attributed to the higher doping level in the case of FIBID, as given by composition measurements obtained with energy-dispersive X-ray microanalysis.
\end{abstract}

Copyright (c) 2009 J. M. De Teresa et al. This is an open access article distributed under the Creative Commons Attribution License, which permits unrestricted use, distribution, and reproduction in any medium, provided the original work is properly cited.

\section{Introduction}

Focused-electron- and focused-ion-beam-induced deposition (from now on, FEBID and FIBID, resp.) of metallic materials is one major application of "dual beam" systems, which integrate electron and ion columns. FEBID and FIBID allow local deposition in the targeted place with controllable lateral size (typical size from $15 \mathrm{~nm}$ to $10 \mu \mathrm{m}$ ) and thickness (typically ranging from $10 \mathrm{~nm}$ to $10 \mu \mathrm{m}$ ). Amongst applications of FEBID and FIBID deposits, one can cite the reparation of optical masks and integrated circuits [1], fabrication of three-dimensional nanostructures $[2,3]$, deposition of protection layers for lamella preparation [4], creation of electronic nanodevices [5], fabrication of nanoelectrodes and nanocontacts [6], transport studies of nanowires $[7,8]$, and deposition of magnetic $[9,10]$ and superconducting materials [11-13].

FEBID and FIBID involve a chemical vapor deposition process that is assisted by electron and ion beams, respectively (for recent review articles, see [14-16]). Roughly speaking, precursor gas molecules containing the material to be deposited flow from an injector toward the substrate and become adsorbed to it. The precursor gas molecules are decomposed by the electron or ion beams, and the material is locally deposited. More in detail, this growth technique is based on three physical/chemical processes. The first one is the substrate-precursor molecule interactions, involving mechanisms such as diffusion, adsorption, and desorption. The second one is the electron/ion-substrate interaction. A 
primary beam of electrons/ions is focused onto the substrate. Some of these electrons collide and are deflected from their initial trajectory whereas other ones undergo inelastic collisions transferring the energy to other electrons/ions. The third one is the electron-precursor molecule interaction. The probability that an electron induces the scission of a bond in a precursor molecule depends on the electron energy and is generally expressed as a cross-section $\left(\mathrm{cm}^{2}\right)$. It depends on the energy of the bonds within the molecule and, in general, electrons with energy of a few $\mathrm{eV}$ are able to produce the molecule dissociation. Besides, two clear deposition regimes have been identified. In the first regime, coined electronlimited regime, the growth is limited by the current density and is independent of the gas flux. In the second regime, coined precursor-limited regime, the growth is limited by the number of molecules arriving at the irradiated area [16].

The physical phenomena involved in the deposition are thus complex, and models trying to explain the experimental results take into account factors such as the electron flux as well as their spatial and energy distribution, the crosssection of the precursor as a function of electron energy, the precursor residence time, the electron-beam-induced heating, etc. An important piece of information has been gained through Monte Carlo simulations [14]. Those simulations have highlighted the important role played by the generated secondary electrons in the deposition rate and also in the spatial distribution and resolution of the nanodeposits.

When the precursor molecules are organic, in general they are not fully decomposed and, together with the metallic component, a carbonaceous matrix is also present, which is relevant for the physical and chemical properties exhibited by the deposit. One of the most used metallic materials in these deposits is $\mathrm{Pt}$, which calls for detailed characterization of the process and the deposited material. In previous studies, the resistivity values of as-grown $\mathrm{Pt}$ nanowires grown with FEBID and FIBID were found to be strongly dependent on the deposition parameters but, in general, the FIBID deposits showed lower resistivity values [17-21]. Different conduction mechanisms have been proposed in these $\mathrm{Pt}$ nanodeposits but a clear picture is not established yet, mainly due to the important role played by the carbonaceous matrix. The low percentage of $\mathrm{Pt}$ in the deposits compared to the residual $\mathrm{C}$ is a handicap for their long-searched metallic behavior.

In the present work, we study the transport properties of Pt by FEBID and FIBID with in-situ real-time control of the deposit resistance by means of electrical microprobes. One advantage of the in-situ electrical characterization compared to the ex-situ one is that spurious effects such as oxidation or contamination under ambient conditions will not play any role in the obtained results. These measurements are complemented with more standard ex-situ transport measurements in a cryostat. In order to give a satisfactory explanation of the observed transport behavior, detailed transmissionelectron-microscopy (TEM) and energy-dispersive X-ray microanalisis (EDX) studies have been performed. The set of results allows us to propose a comprehensive explanation of the differences between Pt nanodeposits grown by FEBID and FIBID.

\section{Experimental}

For the present experiments, a commercial "dual beam" instrument (Nova 200 NanoLab from FEI) has been used. It integrates a $30 \mathrm{kV}$ field-emission electron column and a Gabased $30 \mathrm{kV}$ ion column placed forming $52^{\circ}$ with coincidence point at $5 \mathrm{~mm}$ away from the electron-column pole. For Pt deposition, an automatized gas-injection system (GIS) was used with $\left(\mathrm{CH}_{3}\right)_{3} \mathrm{Pt}\left(\mathrm{CpCH}_{3}\right)$ as the precursor material. The GIS tip was positioned about $150 \mu \mathrm{m}$ away from the region of interest in the $z$ direction and about $50 \mu \mathrm{m}$ away in the $x / y$ direction. The GIS was heated to about $40^{\circ} \mathrm{C}$ for operation, and a 10-minute preheating period was realized before deposition. The real temperature in the GIS can be a bit lower $(\approx 1 \mathrm{~K})$ than the set point [12].

For the investigation of the composition, growth of $\mathrm{Pt}$ by FEBID and FIBID was carried out in $5 \times 5 \mu \mathrm{m}^{2}$ areas on a Si substrate. After waiting for a few minutes in order to allow most of the adsorbed precursor molecules to leave the substrate surface, EDX microanalysis was performed by means of a commercial Oxford INCA 200 EDX setup whose detector is driven in the vicinity of the sample. Prior to each EDX experiment, energy calibration by means of a Co calibration sample was done. The volume per dose has been calculated after doing cross-section inspections of the deposits in order to measure the deposit thickness.

For the electrical measurements of the Pt deposits, we designed experiments where the deposits close a $12 \mu \mathrm{m}$ gap between metal electrodes. The metal $\mathrm{Al}$ electrodes were micropatterned on a $\mathrm{Si}_{3} \mathrm{~N}_{4}(300 \mathrm{~nm}) / \mathrm{Si}$ substrate following these steps: (1) the $\mathrm{Si}_{3} \mathrm{~N}_{4}$ layer was grown with a plasma-enhanced chemical-vapor-deposition equipment (from Sistec Company) on a Si wafer in order to ensure good electrical insulation from the substrate; (2) an optical lithography lift-off process was carried out via an image reversal photoresist and metal deposited with an electronbeam evaporator (from Edwards Company) and finally acetone immersion. The mask design was done so that contact pads for measurements of the electrical resistance in 2-probe as well as 4-probe were available.

Electrical microprobes (from Kleindiek) were used for precise in-situ control of the deposit resistance [22]. These conductive microprobes are connected via a feedthrough to a Keithley 2000 multimeter located out of the dual beam chamber, which allows measuring the deposit resistance by two-probe measurements provided that its value is below $120 \mathrm{M} \Omega$. A two-probe method is used to measure the resistance applying constant current of value $700 \mathrm{nA}$ and continuous monitoring. The lead resistance was only about $13 \Omega$, which guarantees no significant influence on the measured resistance of the deposit, which is at least two orders of magnitude larger as will be shown later. In the currentversus-voltage characterization and in the measurements as a function of temperature, four-probe measurements were performed with a 6220 DC current source-2182A 


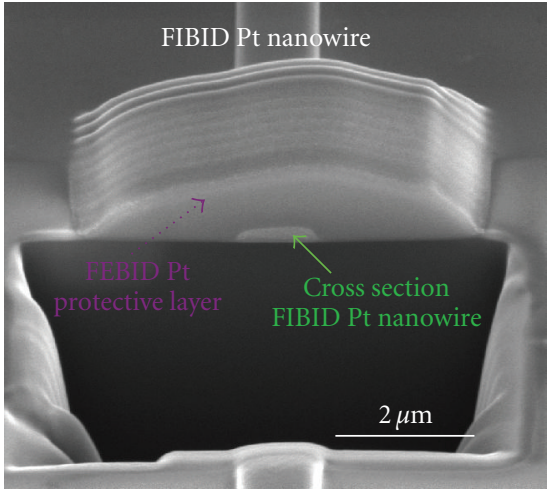

(a)

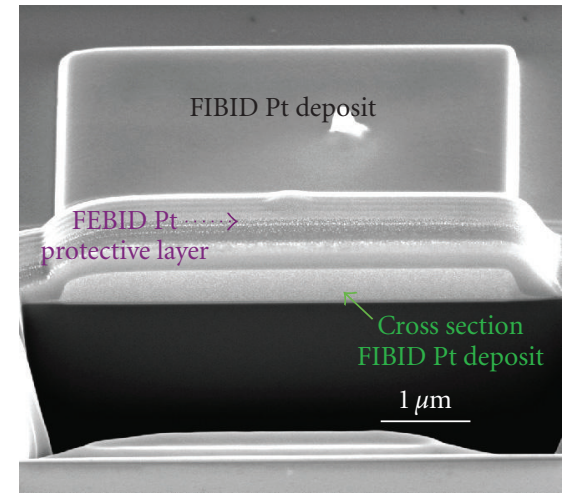

(b)

FIGURE 1: SEM images taken after cross-section experiments performed in (a) a FIBID Pt nanowire and (b) a FIBID Pt square deposit. In both cases, a layer of FEBID Pt is deposited on top of the FIBID deposits before ion milling in order to protect the area to be investigated and to get higher image contrast for reliable thickness measurements.

nanovoltmeter combined Keithley system, which warrants no influence of any contact resistance. For the measurements as a function of temperature, microcontacting to a chip carrier has been performed, and a closed-cycle refrigerator has been used to cool down the sample.

The high-resolution TEM (HRTEM) study was carried out using a Jeol $2010 \mathrm{~F}$ equipment operated at $200 \mathrm{kV}$ (pointto-point resolution $0.19 \mathrm{~nm}$ ) on Pt deposits either grown on $\mathrm{Cu}$ TEM grids with a supporting carbon membrane or by inspection of a typical thin lamella ( $<100 \mathrm{~nm}$ thick) prepared after deposit growth.

\section{Results}

The results presented hereafter have been obtained in Ptbased nanodeposits grown either in the form of nanowires or in the form of square areas. In Figure 1, we show typical cross-section SEM images of these nanodeposits. The standard cross-section method for the imaging of the place of interest involves: (1) the deposition of another material on top of it for protection and high image contrast, (2) FIB milling from the top and cleaning procedure at low current or voltage, (3) sample tilting and SEM imaging.

As can be observed in Figure 1(a), our nanowires are typically a few microns long, one micron wide, and a few tens or hundreds of nanometers thick. The square nanodeposits have typically $\approx 5 \times 5 \mu \mathrm{m}^{2}$ area as shown in Figure $1(\mathrm{~b})$ and thickness of some hundreds of nanometers. The shown nanowire and nanodeposit correspond to Pt FIBID samples which have been covered with a protective Pt FEBID layer for the cross-section evaluation.

3.1. Volume per Dose of the Pt Nanodeposits. In order to compare the transport properties of FEBID and FIBID samples with similar thickness, the volume per dose (in $\mu \mathrm{m}^{3} / \mathrm{nC}$ ) has been first investigated. The procedure to calculate the volume per dose consists in growing the nanodeposits in a $\approx 5 \times 5 \mu \mathrm{m}^{2}$ square area, measuring the thickness by standard cross-section experiments with the assistance of FIB milling and SEM inspection, and calculating the total amount of charge received by the sample. The ratio of the nanodeposit volume to the charge gives the volume per dose.

Pt by FEBID at several incident electron beam energy in the range from $1 \mathrm{kV}$ to $30 \mathrm{kV}$ was performed. At each fixed beam energy, several depositions of size $\approx 5 \times 5 \mu \mathrm{m}^{2}$ have been carried out with five different beam currents in the range $0.4-9.5 \mathrm{nA}$. The electron beam current was found not to have any significant influence on the volume per dose. In Figure 2(a), the volume per dose is represented as a function of the beam energy. Thus, for a fixed beam energy, the average volume-per-dose value of all the investigated currents is represented together with the standard deviation (error bar). In every experiment, the nanodeposit thickness is evaluated by the standard cross-section method. It can be noticed that the volume per dose decreases as a function of the beam energy, as previously found for FEBID of $\mathrm{Pt}$ $[23,24]$. The decrease is rather fast up to $5 \mathrm{kV}$ and flattens above $15 \mathrm{kV}$ as previously found. At $30 \mathrm{kV}$, the volume per dose is four times lower than at $1 \mathrm{kV}$. This dependence can be explained by the decrease in the amount of secondary electrons generated in the substrate and reaching the sample surface $[14-16,23,24]$. The deposition rate, $R$, is governed by the formula

$$
R=\int_{0}^{E_{0}} f(E) \sigma_{\text {diss }}(E) N d E
$$

where $E_{0}$ is the energy of the primary electrons, $f(E)$ and $\sigma_{\text {diss }}(E)$ are, respectively, the flux of electrons and the electron-impact dissociation cross-section at given energy, and $N$ is the molecular density on the surface. $\sigma_{\text {diss }}(E)$ is experimentally found to show a maximum at a certain energy in the range of a few tens of eV (see the example of $\mathrm{C}_{2} \mathrm{H}_{5}$ in [14], with maximum in $\sigma_{\text {diss }}$ at $18 \mathrm{eV}$ ). On the other hand, the distribution of secondary electrons generated by the incident electron beam is maximum in the same range of energy, which favors that secondary electrons contribute largely to the molecule precursor dissociation. As the secondary 


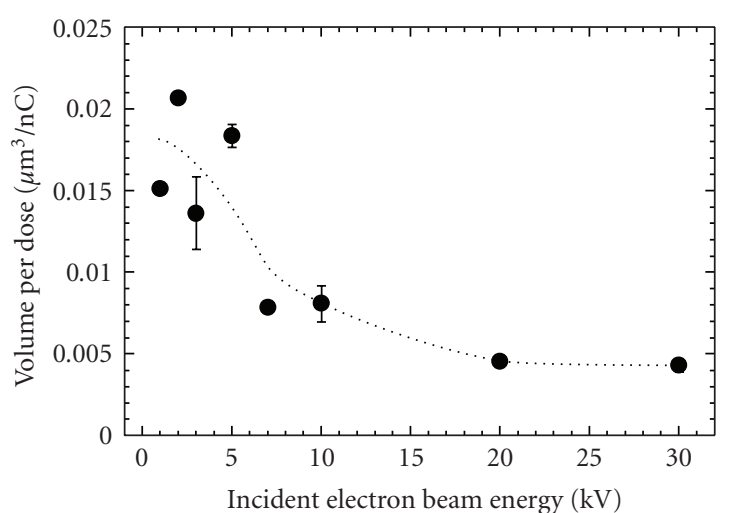

(a) FEBID

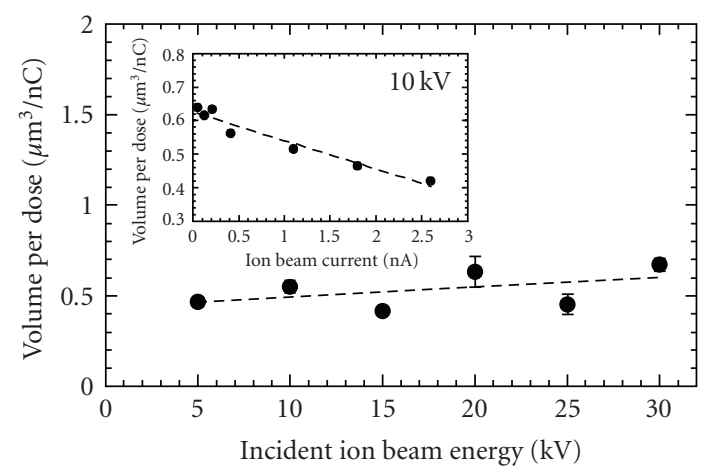

(b) FIBID

FIgURe 2: (a) Average volume per dose versus incident electron beam energy in the investigated Pt deposits by FEBID; (b) average volume per dose versus incident electron beam energy in the investigated Pt deposits by FIBID. The inset shows the volume per dose versus ion beam current in the investigated FIBID deposits at fixed incident beam energy of $10 \mathrm{kV}$.

electron yield decreases with the incident electron beam energy, the diminishment of deposition rate with increasing beam energy is naturally explained.

Pt by FIBID at several incident ion beam energies in the range from $5 \mathrm{kV}$ to $30 \mathrm{kV}$ was performed with beam currents from $44 \mathrm{pA}$ to $2.6 \mathrm{nA}$. In sharp contrast with Pt by FEBID, in Pt by FIBID the beam current modifies the volume per dose at fixed incident beam energy. Figure 2(b) indicates that the volume per dose slightly increases as a function of the beam energy. This fact can be explained by a higher amount of secondary electrons being generated at higher beam energy and being able to reach the sample surface. In contrast to electrons, the Ga ions, even at high energy of $30 \mathrm{kV}$, are stopped in about $50 \mathrm{~nm}$ below the sample surface [23], which allows the generated secondary electrons to reach more easily the sample surface. As shown in the inset of Figure 1(b) for deposits at $10 \mathrm{kV}$, the volume per dose decreases as a function of the beam current, which is explained by the lack of full refreshment of the precursor molecules adsorbed to the sample surface at high beam currents. Thus, a $50 \%$ higher volume per dose can be obtained at $50 \mathrm{pA}$ compared to $2.6 \mathrm{nA}$. This decrease of the volume per dose with the beam current is also observed in deposits performed at different

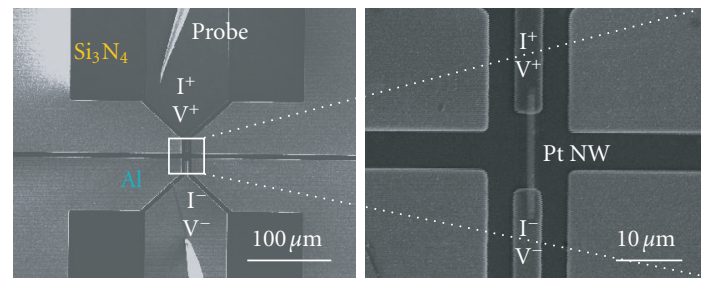

(a)

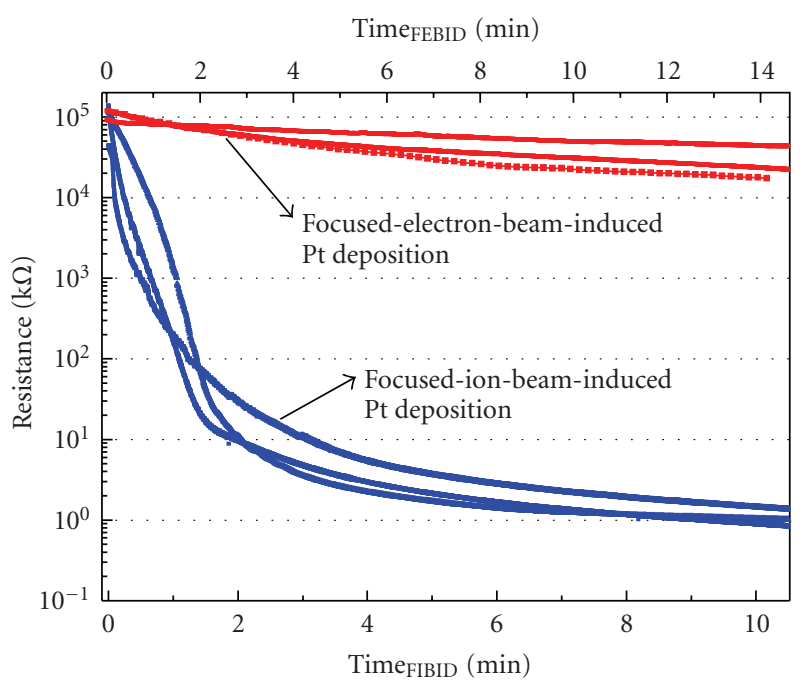

(b)

FIgure 3: The image on the left top is a SEM picture of the electrical pads patterned by optical lithography together with the two microprobes contacted for in-situ control of the deposit resistance. The image on the right top is a SEM picture of one typical deposit closing the gap between the contact electrodes for the measurement of the resistance. The shown results of resistance versus time correspond to the comparison between several $\mathrm{Pt}$ samples grown by FEBID and FIBID. Resistance monitorization starts after 6.3 minutes in the case of the FEBID process and 1.5 minutes in the case of the FIBID process. The final deposit thickness is approximately the same $(160 \mathrm{~nm})$ in all cases (see text for details). For the sake of clarity, zero time is the starting point of the resistance monitorization in all cases.

beam energies (from $5 \mathrm{kV}$ to $30 \mathrm{kV}$, results not shown). As a further example, at $5 \mathrm{kV}$ it is obtained that the volume per dose decreases from $0.52 \mu \mathrm{m}^{3} / \mathrm{nC}$ to $0.39 \mu \mathrm{m}^{3} / \mathrm{nC}$ with the increase of the ion beam current from $70 \mathrm{pA}$ to $1 \mathrm{nA}$. These results show that with those large ion beam currents, the growth belongs to the precursor-limited regime, in sharp contrast to the case of FEBID of Pt described above.

\subsection{In-Situ Control of the Electrical Resistance during Depo-} sition and Comparison of the Transport Properties. The possibility of in-situ control of the electrical resistance during deposition, which is demonstrated in Figure 3, is very interesting and has been previously reported with different approaches in $[12,25]$. In our case, by careful initial positioning of the electrical microprobes with respect to the contact electrodes and final adjustments with the help of 
the electron and ion beams imaging, it is possible to realize in-situ good electrical contacts between the probes and the contact electrodes [22]. Thus, if we locate the electrical microprobes on a metallic electrode, we measure $13 \Omega$, which is the specified lead resistance. In our targeted experiments, the electrodes are separated by a $12 \mu \mathrm{m}$ gap, which is closed with the FEBID or FIBID deposits (with size $1 \times 20 \mu \mathrm{m}^{2}$ ). After certain time, the deposit resistance is below $120 \mathrm{M} \Omega$, and we start to monitor continuously how the resistance decreases as the deposition time goes on.

In Figure 3 we compare Pt samples by FEBID and FIBID with similar thickness in order to give evidence for the success in the application of the method as well as the strong difference in the resistance of the deposits. In all cases a multiscan (several beam passes) process is used. Three FEBID and FIBID deposits are shown to illustrate the reproducibility of the results. In the case of the shown FEBID deposits, we have used the conditions: beam current = $0.54 \mathrm{nA}$, beam energy $=10 \mathrm{kV}$, and dwell time $=1 \mu \mathrm{s}$. After 6.3 minutes, the resistance reaches $120 \mathrm{M} \Omega$, and we start the resistance monitorization. After additional deposition time of 14.6 minutes, the final resistance is found to be $23.5 \mathrm{M} \Omega$. In the case of the FIBID deposits shown, we have used the conditions: beam current $=10 \mathrm{pA}$, beam energy $=30 \mathrm{kV}$, and dwell time $=200 \mathrm{~ns}$. After 1.5 minutes, the resistance reaches $120 \mathrm{M} \Omega$, and we start the resistance monitorization. After additional deposition time of 10.7 minutes, the final resistance is found to be $1 \mathrm{k} \Omega$. Even though the final thickness of all the deposits is roughly the same $(\sim 160 \mathrm{~nm})$, the resistance is four orders of magnitude lower for Pt by FIBID. For these nanodeposits of thickness $160 \mathrm{~nm}$, the resistivity value in the case of the Pt by FEBID is about $10^{7} \mu \Omega \mathrm{cm}$ whereas it is about $800 \mu \Omega \mathrm{cm}$ in the case of Pt by FIBID, in any case much higher than for bulk $\mathrm{Pt}, 10.8 \mu \Omega \mathrm{cm}$.

In a homogeneous system with a certain resitivity, $\rho$, one expects Ohm's law to hold

$$
R=\rho \frac{l}{S} \propto \frac{1}{\text { thickness }}
$$

Assuming that the nanodeposit thickness is proportional to the deposition time, one can represent the resistance versus the inverse of the thickness in order to investigate if this behavior occurs in the FEBID and FIBID nanodeposits. This representation is shown in Figure 4 for selected FEBID and FIBID nanodeposits. In the case of the FEBID nanodeposit, such scaling is not obtained but a slight decrease of the resistivity with thickness. In the case of the FIBID nanodeposit, the effects are dramatic. It is clearly observed that the scaling of the resistance with the inverse of thickness only occurs for thickness above $\sim 100 \mathrm{~nm}$. This means that the nanodeposit resistivity is changing rapidly during the first $\sim 100 \mathrm{~nm}$ and finally stabilizes at about $800 \mu \Omega \mathrm{cm}$. A similar behavior has been observed by Spoddig et al. in Pd-based nanowires grown with FIBID [12]. It can be speculated that as the nanodeposition starts and proceeds, the nanodeposit composition changes as a function of the thickness due to the "changing substrate" seen by the incoming ions. As we have described in Section 1, the interactions between the precursor molecules and the substrate as well as the incoming

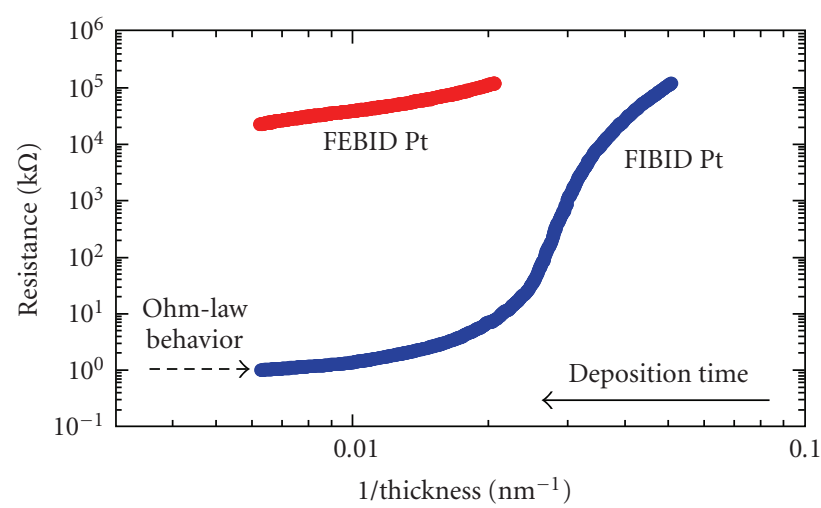

FIgure 4: Representation of the resistance as a function of the inverse of thickness for one selected FEBID Pt nanowire and one selected FIBID Pt nanowire, which illustrates that the constant value expected if the Ohm-law applies only occurs for the FIBID Pt deposits above $\approx 100 \mathrm{~nm}$.

ions with the substrate is crucial for the physical phenomena involved in the nanodeposition, and these interactions will change with thickness during the first tens of nanometers. A focused work on this interesting issue is currently in progress.

In Figure 5, again in-situ, we compare the four-probe current-versus-voltage dependence after finishing the growth of one FEBID and one FIBID Pt deposit of similar thickness to those of Figure 3. As can be seen in the SEM image of Figure 5, it is possible to place the four electrical microprobes and the GIS in the area of interest. These measurements have the advantage that any lead resistance or contact resistance is eliminated, and the intrinsic resistance of the nanowire is measured. Whereas the FIBID deposit, with resistance about $1 \mathrm{k} \Omega(\rho \approx 800 \mu \Omega \mathrm{cm})$, shows a linear dependence in the studied voltage range, the FEBID deposit, with resistance about $50 \mathrm{M} \Omega\left(\rho \approx 10^{7} \mu \Omega \mathrm{cm}\right)$, shows a nonlinear dependence. The differences between both types of behavior lie on the different content of the nanodeposits as will be demonstrated later. The linear behavior of the FIBID $\mathrm{Pt}$ is the expected response for a metallic or quasimetallic system. On the other hand, the non-linear behavior observed in the FEBID Pt deposit can be explained on the basis of the relevant role played by the semiconducting carbonaceous matrix. Interestingly, in the inset of Figure 5(b), the conductance of the FEBID Pt deposit is represented as a function of the applied voltage. It is observed that the conductance decreases up to $\approx 0.6 \mathrm{~V}$ and then starts to increase for higher voltages. We interpret this result within the theoretical framework developed by Van Lien and Shklovskii [26], further studied with Monte Carlo simulations [27], and experimentally observed in doped silicon $[28,29]$. Basically, in semiconducting systems with conduction via impurities the application of electrical field can block the hopping of electrons opposite to the electrical field direction, reducing first the conductivity up to a certain electric field value, where it starts increasing again due to hopping to the localizedstates band tail or the unlocalized states band. A deeper discussion on this point will be elaborated in Section 4. 


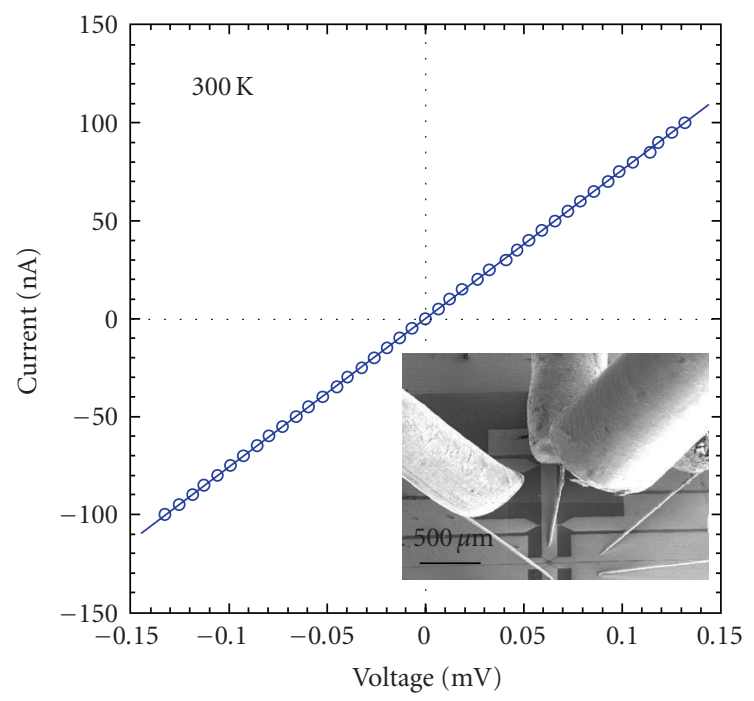

(a) FIBID Pt $300 \mathrm{~K}$

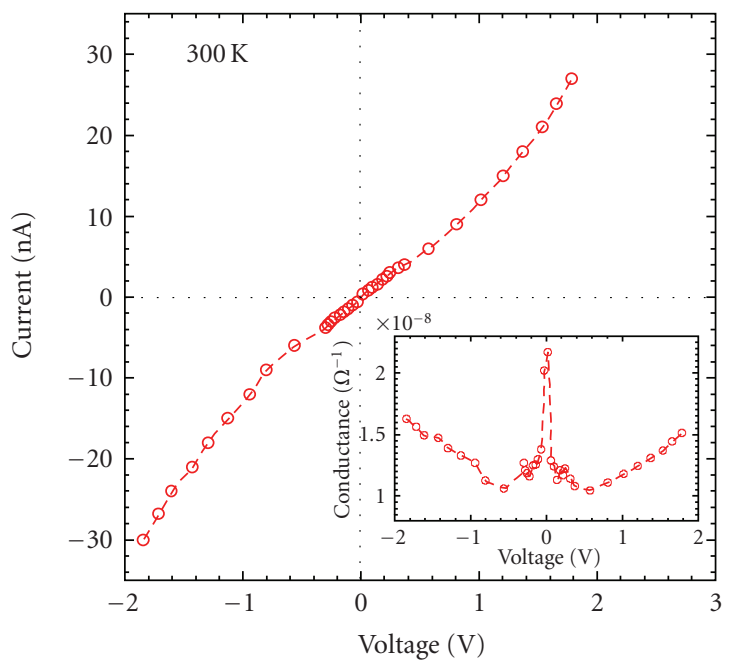

(b) FEBID Pt $300 \mathrm{~K}$

FIGURE 5: In-situ four-probe current-versus-voltage measurements of FEBID and FIBID Pt deposits similar to those of Figure 3. (a) The measurements in the FIBID Pt nanowire indicate linear dependence between current and applied voltage. The inset shows a SEM picture where the 4 electrical microprobes and the Pt-based gas injector can be located in the vicinity of the area of interest for the realization of the current-versus-voltage measurements. (b) whereas those in the FEBID Pt nanowire indicate non-linear dependence. The inset shows the anomalous dependence of conductance versus voltage, which is explained in the text.

We have subsequently studied the ex-situ transport properties of the deposits by four-probe measurements in a cryostat. This characterization is useful for two reasons. First, it provides additional information on the conduction mechanism. Second, if these nanodeposits are going to be used at low temperatures, their transport properties must be known. In Figure 6 the dependence of the resistance with temperature for the same FIBID and FEBID deposits as in Figure 5 is shown. Whereas the resistance of the

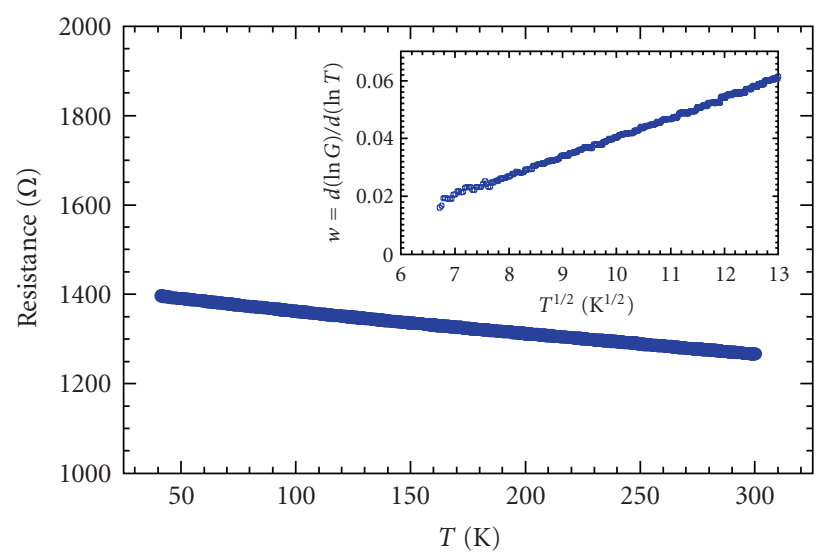

(a) FIBID Pt

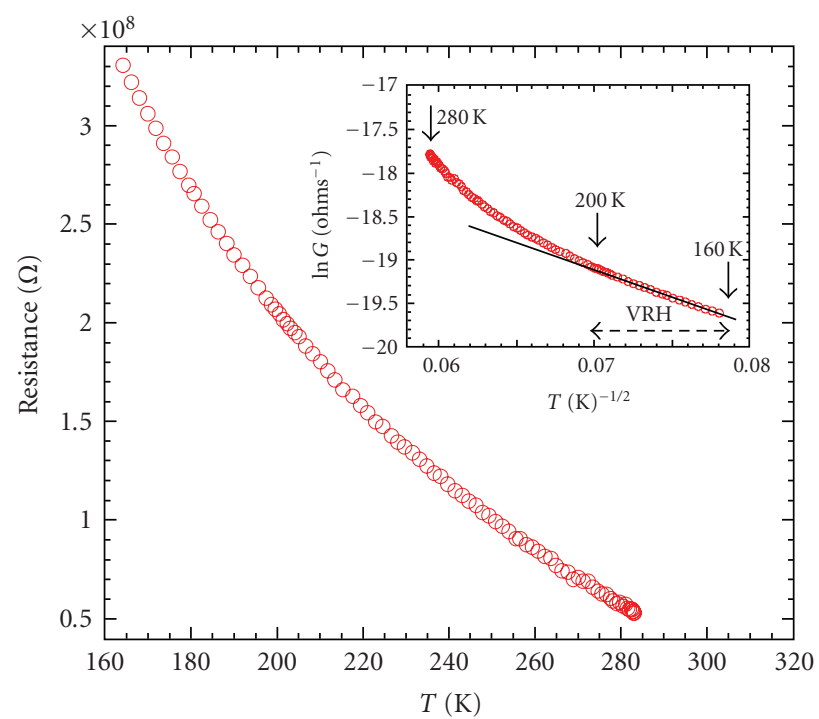

(b) FEBID Pt

Figure 6: (a) Temperature variation of the resistance of a FIBID Pt nanowire showing a quite flat dependence. The inset shows the analysis of data in the form of $w$ versus $T^{1 / 2}$. (b) Temperature variation of the resistance of a FEBID Pt nanowire, showing a semiconducting dependence. The inset shows the analysis of data in the form of $\ln (G)$ versus $T^{-1 / 2}$ (see text for details).

FIBID deposit only increases very slightly with decreasing temperature (a factor of 1.1 at $150 \mathrm{~K}$ compared to $300 \mathrm{~K}$ ), the FEBID deposit shows a strong semiconductor behavior, and at $T=150 \mathrm{~K}$ the resistance is of the order of $1 \mathrm{G} \Omega$, the maximum resistance that our experimental setup can measure (a factor of 5.5 compared to $300 \mathrm{~K}$ ). Further understanding of the conduction mechanisms has been obtained by the performed data analysis.

In the case of the FIBID Pt deposit, and in order to study if the temperature dependence of the resistance is compatible with a metallic or an insulating material, we use the methodology proposed by Möbius et al. [30]. As shown in the inset of Figure $6(\mathrm{a}), w=d(\ln G) / d(\ln T)$ is represented as a function of $T^{1 / 2}$. According to [30], if $w$ tends to zero at low temperature, it means that the compound behaves 
as a metal. In fact this is the tendency observed in our measurements even though further measurements at very low temperatures would be required to fully determine this point. As a consequence, the FIBID Pt deposit has metallic (or quasimetallic) conduction as would be expected if a semiconductor matrix is highly doped so that an impurity band or a percolation network builds up.

In the case of the FEBID Pt deposit, the temperature dependence of the resistance is compatible with hopping conduction in a band of localized states inside a semiconductor matrix [31]. In the inset of Figure 6(b), the representation of $\ln (G)$ versus $T^{1 / 2}$ indicates that below $200 \mathrm{~K}$ the conduction mechanism fits the model of variable-range-hopping with Coulomb interaction developed by Efros and Shklovskii [32], where $R \propto R_{0} \exp \left(T_{0} / T\right)^{1 / 2}$. The same temperature dependence has been previously found in FEBID Pt deposits by Tsukatani et al. [33]. Above $200 \mathrm{~K}$, the thermal energy is high enough to allow other electronic processes to be involved such as hopping via thermal activation, leading to a deviation from the $T^{-1 / 2}$ law.

These results stress the different transport behavior of $\mathrm{Pt}$ by FEBID and FIBID. By changing process parameters such as the beam energy or current within accessible ranges, we do not find significant differences in the transport results, the FEBID of Pt always being several orders of magnitude more resistive than the FIBID one. In order to investigate possible origins of such strong difference, we have carried out further experiments regarding the microstructure and composition of the nanodeposits.

\subsection{Microstructure Investigated by High-Resolution Transmis-} sion Electron Microscopy. HRTEM experiments of FEBID and FIBID Pt deposits grown at several energies and beam currents have been performed in order to investigate any difference in the microstructure. Two approaches have been followed. First, several lamellae were prepared out of the deposits grown on Si substrates. Second, the FEBID and FIBID Pt deposits (thickness in the range 20-50 nm) were directly grown on $\mathrm{Cu}$ TEM grids with a supporting carbon membrane. Whereas the first approach produces results representative of the samples studied in the transport experiments, the second approach gives more clear TEM images but are grown on a different substrate from the samples studied in transport experiments. Both studies are consequently shown in order to show that both types of results are compatible.

From the EDX results to be shown in the next subsection, we know that the main constituents of the deposits are $\mathrm{C}$ and $\mathrm{Pt}$. As Pt is a high-atomic-number element compared to C, in HRTEM images it is expected that the Pt-rich areas appear darker than the C-rich areas. However, the interpretation of the TEM images can be tricky because the obtained 2D image contains information of all the way traveled by the electrons along the sample thickness $(50-100 \mathrm{~nm})$. This is why it is not straightforward to reach conclusions just from the black/white contrast observed in the images, especially because the grains are small compared to the sample thickness, and the transmitted electrons can meet several grains in their path across the sample. The TEM images obtained in the lamellae fabricated from FIBID and FEBID Pt deposits are shown in Figure 7. The observed black/white contrast is due to the inhomogeneous distribution of $\mathrm{Pt}$, and $\mathrm{C}$ in the deposits as previously reported [17-21] and in principle can be roughly assigned to Pt nanoparticles with size below $5 \mathrm{~nm}$ immersed in the amorphous carbon matrix. However, the lack of clear observation of atomic planes is a drawback for the correct interpretation of the images. On the other hand, the observation of atomic planes in the deposits grown directly on the $\mathrm{Cu}$ grids permits an unambiguous interpretation of the images. As can be observed in Figure 8, the FEBID and FIBID Pt deposits consist of ellipsoidal crystalline Pt grains embedded in an amorphous carbonaceous matrix. The crystalline Pt grains are easily identified due to the clear observation of Pt atomic planes with the expected planar distances. Thus, fast-Fourier transforms of the images give as a result the presence of diffraction spots corresponding to the planar distances of $0.2263 \mathrm{~nm}, 0.1960 \mathrm{~nm}, 0.1386 \mathrm{~nm}$, and $0.1132 \mathrm{~nm}$, which correspond, respectively, to the (111), (200), (202), and (222) atomic planes of $\mathrm{fcc} \mathrm{Pt}$, with lattice parameter of $0.3924 \mathrm{~nm}$. An average size of Pt crystallites has been obtained from the measurement of about 50 individual grains in each image through the counting of the number of atomic planes. It is found that no matter the used beam energy and current are, in all the FEBID and FIBID deposits the average Pt crystallite size is about $3.2 \pm 0.8 \mathrm{~nm}$. In $W$ nanodeposits by FEBID, it has been found that by using very high beam energies $(>200 \mathrm{kV})$ it is possible to modify the nanocrystallite size [34]. To the best of our knowledge, such high beam energy has not been used to create Pt by FEBID.

Thus, from these HRTEM images, the microstructure of the FEBID and FIBID Pt deposits is similar. We would like to stress that the microstructures observed in both, the lamellae and the TEM grids, are qualitatively similar. However, for quantitative analysis only the deposits on the TEM grids can be reliably used.

3.4. Composition of the Pt Nanodeposits. First, we checked that the EDX results obtained on nanowires was equivalent to those obtained on square nanodeposits of size $\approx 5 \times$ $5 \mu \mathrm{m}^{2}$, which are preferred for the EDX experiments. First, we analyzed a FEBID Pt nanodeposit carried out at $10 \mathrm{kV}$ incident beam energy. The nanowire composition found by $\mathrm{EDX}$ was $\mathrm{C}(\%)=87.81 \pm 0.21, \mathrm{Pt}(\%)=12.19 \pm 0.21$ whereas the square area composition was found to be $\mathrm{C}(\%)=85.46 \pm 0.69, \mathrm{Pt}(\%)=13.47 \pm 0.40$. Afterwards, $\mathrm{a}$ FIBID Pt nanodeposit carried out at $30 \mathrm{kV}$ incident beam energy was analyzed. The nanowire composition found by $\mathrm{EDX}$ was $\mathrm{C}(\%)=71.80 \pm 0.22, \mathrm{Pt}(\%)=17.40 \pm 0.22$, and $\mathrm{Ga}(\%)=10.80 \pm 0.21$, whereas the square area composition was found to be $\mathrm{C}(\%)=72.84 \pm 0.52, \mathrm{Pt}(\%)=16.67 \pm 0.26$, and $\mathrm{Ga}(\%)=10.50 \pm 0.12$. Thus, we concluded that the composition could be extracted reliably from the EDX results on the square samples.

In Figure 9(a) the average Pt content of FEBID of $\mathrm{Pt}$, as obtained with EDX measurements, is shown as a 


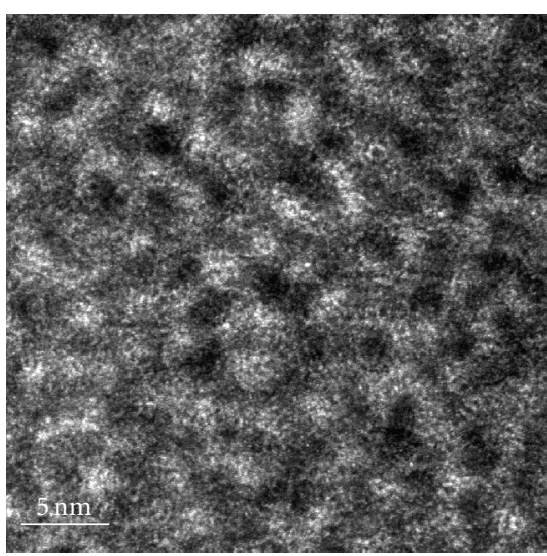

(a) FIBID Pt deposit

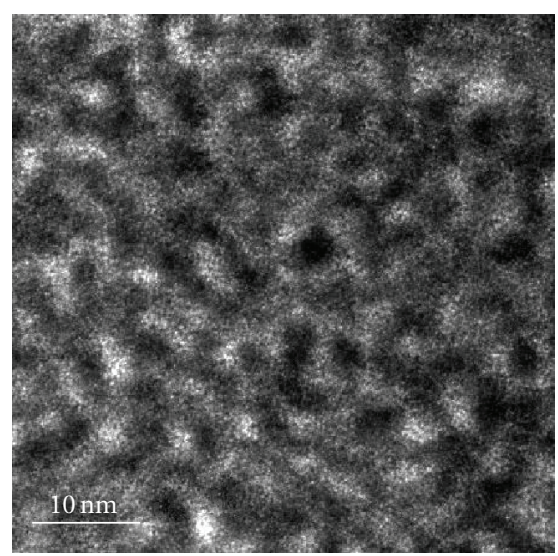

(b) FEBID Pt deposit

FIGURE 7: HRTEM images obtained out of lamellae fabricated respectively from a Pt nanodeposit by (a) FIBID and (b) FEBID, in both cases grown at $5 \mathrm{kV}$ beam energy.

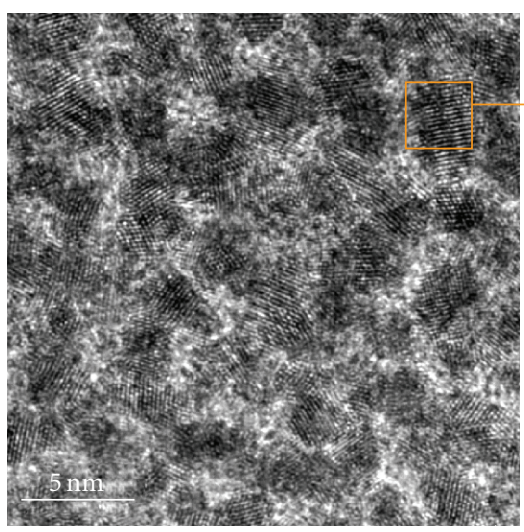

(a) FIBID Pt deposit
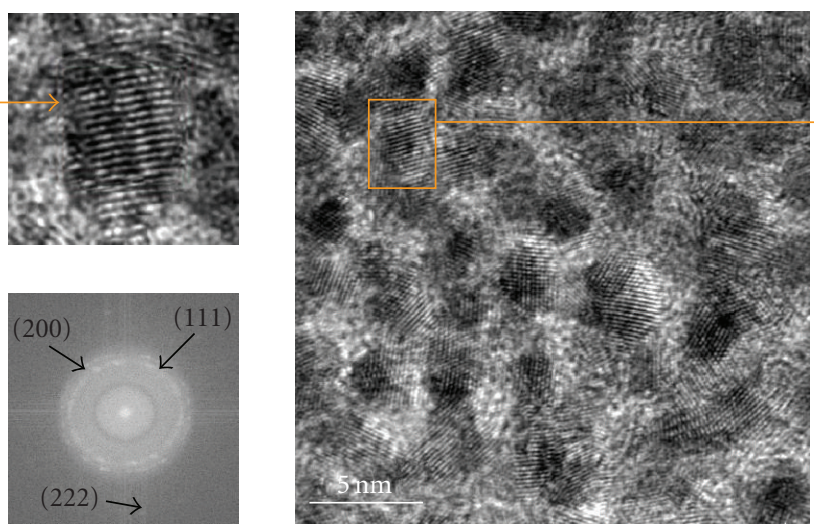

(b) FEBID Pt deposit
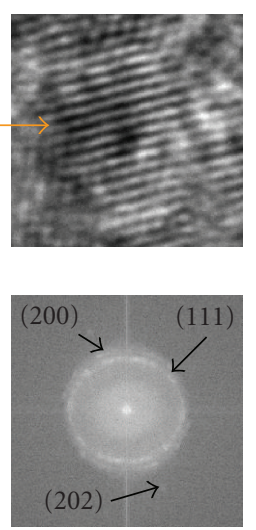

FIgURE 8: HRTEM images of a Pt nanodeposit by (a) FIBID and (b) FEBID, in both cases grown at $30 \mathrm{kV}$ beam energy on top of a TEM $\mathrm{Cu}$ grid covered with a thin supporting holey carbon membrane. One Pt grain has been selected in each case for magnification and clear observation of the corresponding atomic planes. The Fast-Fourier-Transform of the full image gives diffraction spots that correspond to the (200), (111), (222), and (202) atomic planes of fcc Pt.

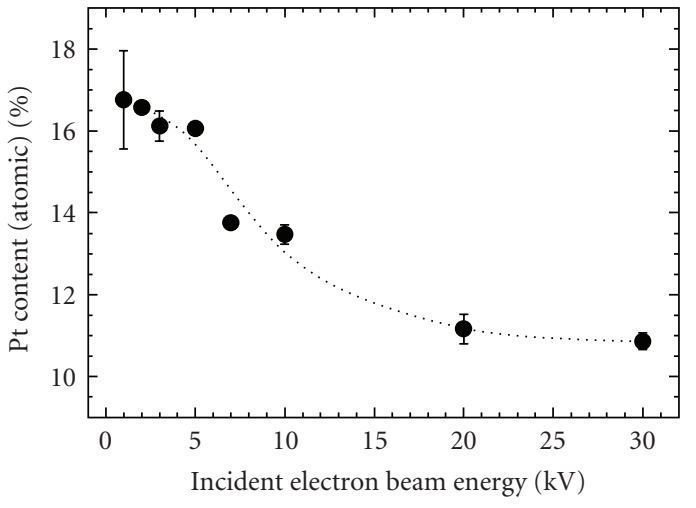

(a) FEBID

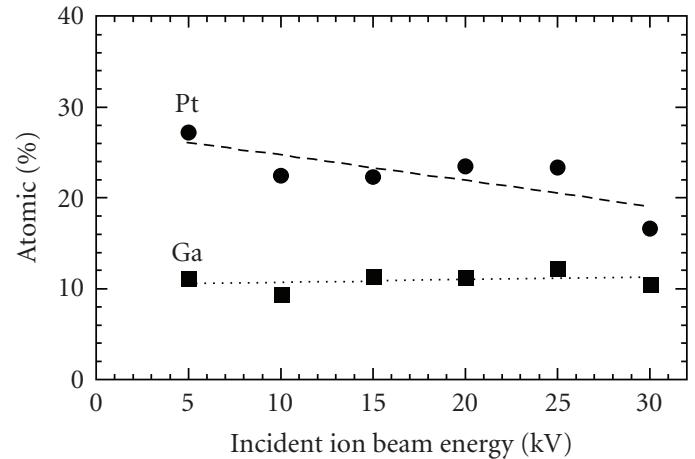

(b) FIBID

Figure 9: Average Pt atomic content (measured by EDX) versus the incident beam energy used for the growth in the investigated (a) FEBID deposits and (b) FIBID deposits. In this last case the Ga content is also shown. An incident electron beam energy of $10 \mathrm{kV}$ was always used for the EDX experiments. 
function of the beam energy used for the growth of the nanodeposit. The nanodeposit thickness is about the same as that of the nanowires $(\approx 160 \mathrm{~nm})$. The incident electron beam energy for the EDX measurement was always $10 \mathrm{kV}$. As the growth beam energy increases, the Pt content is found to decrease from $17 \%$ down to $11 \%$. Thus, the C content in the deposit (the main remaining part, more than $80 \%)$ will be dominant. We did not find any significant change of the deposition content as a function of the beam current. The decrease of Pt content with the beam energy can be due to the lower amount of secondary electrons produced in the substrate reaching the sample surface and being available for precursor gas molecule decomposition. However, the interplay among primary electrons, secondary electrons, and backscattered electrons in the dissociation of the precursor molecules brings about a complex scenario that calls for focused experiments and simulations to provide a more complete picture [14-16]. Regarding the transport properties, it becomes clear that the carbonaceous matrix (more than $80 \%$ in any case) will play a crucial role in the FEBID of Pt.

In Figure 9 (b) the obtained average Pt and Ga content in FIBID of $\mathrm{Pt}$ is shown as a function of the growth beam energy. Whereas the Ga content is roughly constant around $10 \%$, the $\mathrm{Pt}$ content is found to decrease from $27 \%$ down to $17 \%$. The $\mathrm{C}$ content in the deposit is still significant (more than 60\%) but the amount of Pt plus $\mathrm{Ga}$ is now relatively high, which, as discussed in the next section, can be responsible for the observed transport properties.

\section{Discussion}

We argue hereafter that the obtained results can be explained taking into account the important role played by the carbonaceous matrix together with the Pt inclusions, and Gaion implantation in the case of the FIBID. Strong similarities exist between these results and those obtained previously in doped amorphous semiconductors [31]. In those studies, it is typically observed that as the doping level and disorder increase, there appear impurity states in the vicinity of the Fermi level as well as a band tail of localized states separated from the band of unlocalized states at the mobility edge. If the doping level is low, the conduction mechanism at low temperature is carried out via hopping amongst impurity states and in the band tail of localized states, the compound remaining insulating at the lowest temperatures. If the doping level and disorder are high enough, the impurity states can form a band at low temperature allowing metallic (or quasimetallic) conduction, and the situation can be mapped onto a problem of percolation theory of conducting networks [35].

In these Pt nanodeposits, the matrix is amorphous carbon, which is known to behave as a semiconductor with a bandgap of about $2 \mathrm{eV}[36,37]$. Disorder and impurity states arise from the Pt grains (with random position and a certain size distribution), from Ga implantation, and from defects created in the carbonaceous matrix. Pure metallic behavior as a function of temperature is only expected in systems with percolation of the metallic inclusions, which will occur in nanodeposits with high metallic concentration, as in the FIBID Pt deposits. This is first supported by the room-temperature conductivity value found in our FIBID Pt deposits, $\sigma=1250 \Omega^{-1} \mathrm{~cm}^{-1}$, which is around the minimum metallic conductivity value reported by Mott and Davis [31]. The linear current-versus-voltage dependence, the quite flat temperature dependence of the resistivity, and the observed tendency of $w$ to zero at low temperature further support this hypothesis. Similar behavior has been observed, for example, in the doping of amorphous semiconducting Ge with Fe $[31,38]$ and in the doping of amorphous carbon films with I [39]. The nonlinearity observed in [15] in Pt FIBID nanowires with room-temperature resistance of about $20 \mathrm{k} \Omega$ is not inconsistent with our results. A detailed study devoted to explain the temperature dependence of resistance and the current-versus-voltage behavior for FIBID Pt deposits as a function of thickness is in progress and will be published elsewhere.

By contrast, the FEBID Pt deposits will have lower disorder and impurity states due to the smaller amount of Pt grains, the absence of Ga implantation, and the lower degree of disorder caused by electrons compared to ions. Thus, the conduction mechanism in such compounds is the typical one of a low-doped amorphous semiconductor, with a low value of room-temperature conductance, $\sigma=$ $0.1 \Omega^{-1} \mathrm{~cm}^{-1}$, conduction via hopping, and strong increase of resistance at low temperatures. Specially interesting is the observed non-linear current-versus-voltage dependence. In general, in the hopping conduction in semiconductors, an increase in the conductance with the voltage occurs [40]. However, in certain cases, as those reported in [2629], a negative differential conductivity is observed. It was reported in situations with doping level in the order of $10^{16}-10^{17} \mathrm{~cm}^{-3}$, where conduction takes place at low voltage via the localized impurity states. The initial decrease in the conductivity is ascribed to the charge trapping in "dead ends" of the hopping network because the electrons cannot hop in opposite direction to that of the applied electric field. With further increase of applied voltage, the band tail of localized states and the band of unlocalized states above the mobility edge start to be involved in the conduction mechanism and the conductivity increases. This scenario can explain the behavior shown in the inset of Figure 5(b).

\section{Conclusions and Outlook}

We have accomplished a study of the growth of Pt by FEBID and FIBID in a dual-beam equipment, focusing on the difference in the transport properties of the asgrown materials. Owing to the real-time control of the electrical resistance of the growing deposits by means of electrical microprobes contacting metallic pads in a circuit prepatterned by optical lithography, the resistivity of such deposits has been determined without any influence of 
extrinsic effects such as undesired oxidation or contamination. In the range of deposit thickness studied, these measurements indicate that Pt by FIBID is four orders of magnitude more conductive than the FEBID ones. Even in the case of the FIBID of Pt, the resistivity is significantly higher than in bulk Pt (around a factor of 100). Insitu current-versus-voltage measurements on deposits with thickness in the range of $160 \mathrm{~nm}$ indicate that whereas the FEBID Pt ones show non-linear behavior, the FIBID Pt ones show linear behavior at room temperature. Moreover, the resistance of FEBID deposits increases strongly with decreasing temperature in sharp contrast to a smooth dependence in the case of the FIBID ones. These results have been explained within the well-established theoretical framework developed for doped amorphous semiconductors.

As determined by means of HRTEM experiments, the microstructure of both, the FIBID and FEBID deposits, consists of small crystalline Pt grains (average size about $3 \mathrm{~nm}$ ) embedded in an amorphous carbonaceous matrix. A scenario has been proposed where the amorphous carbonaceous matrix is semiconducting and the Pt inclusions (and Ga ions in the case of FIBID) act as impurities and give rise to disorder, providing available states inside the gap either as impurity states or a band tail of localized states. As measured by means of EDX, the amount of dopants in the case of the FIBID nanodeposits is significantly larger than in the case of the FEBID ones (roughly $30-40 \%$ versus $10-20 \%$, resp.), which would explain the difference in the resistivity of both types of deposit.

The obtained results clarify the reason for the difference of resistivity of as-grown FEBID and FIBID of Pt. It is suggested that the conduction is governed by the carbonaceous matrix modulated by the presence of $\mathrm{Pt}$ and $\mathrm{Ga}$. In the case of FIBID of $\mathrm{Pt}$, the nanodeposit is in the verge of metallic percolation showing metallic (or quasimetallic) conduction. However, the FEBID Pt deposits are far from metallic conduction, showing features typical of doped amorphous semiconductors.

Some positive side effects of the presented methodology can be advanced. First, it will be possible to study the deposit growth mechanisms by tracking the time dependence of the electrical resistance of the deposits. Second, the correlation between the measured in-situ and ex-situ electrical resistance of the deposits can be tackled. Our methodology also allows fast studies of how the deposit resistance depends on some deposition parameter (energy, current, gas flow, substrate temperature, etc.), which can be useful for optimized resistance values in a wide range of applications. Besides, Pt nanodeposits have been used to contact nanowires and nanoparticles in order to study locally the electrical transport of such nanostructures [7, 8]. The results of our work indicate that Pt by FIBID seems a better choice for getting lower contact resistance and, as a consequence, less noisy and more reliable transport measurements. We hope that our work will foster further studies aiming to understand the fascinating properties of metallic deposits induced by ion and electron beams, with application in several domains in nanotechnology.

\section{Acknowledgments}

Financial support by Spanish Ministry of Science (through projects MAT2005-05565-C02 and MAT2008-06567-C02, including FEDER funding), and the Aragon Regional Government and experimental help and discussions with Dr. J. Arbiol (Serveis Cientifico-Tecnics, Universitat de Barcelona), Dr. J. Sesé, Dr. A. Ibarra, and L. Serrano are acknowledged.

\section{References}

[1] S. Matsui and Y. Ochiai, "Focused ion beam applications to solid state devices," Nanotechnology, vol. 7, no. 3, pp. 247-258, 1996.

[2] S. Reyntjens and R. Puers, "Focused ion beam induced deposition: fabrication of three-dimensional microstructures and Young's modulus of the deposited material," Journal of Micromechanics and Microengineering, vol. 10, no. 2, pp. 181$188,2000$.

[3] W. Li and P. A. Warburton, "Low-current focused-ion-beam induced deposition of three-dimensional tungsten nanoscale conductors," Nanotechnology, vol. 18, no. 48, Article ID 485305, 5 pages, 2007.

[4] L. A. Giannuzzi and F. A. Stevie, Introduction to Focused Ion Beams, Springer, Boston, Mass, USA, 2005.

[5] A. J. De Marco and J. Melngailis, "Maskless fabrication of JFETs via focused ion beams," Solid-State Electronics, vol. 48, no. 10-11, pp. 1833-1836, 2004.

[6] G. C. Gazzadi and S. Frabboni, "Fabrication of $5 \mathrm{~nm}$ gap pillar electrodes by electron-beam Pt deposition," Journal of Vacuum Science and Technology B, vol. 23, no. 2, pp. L1-L3, 2005.

[7] F. Hernández-Ramírez, J. Rodríguez, O. Casals, et al., "Characterization of metal-oxide nanosensors fabricated with focused ion beam (FIB)," Sensors and Actuators B, vol. 118, no. 1-2, pp. 198-203, 2006.

[8] A. Romano-Rodríguez and F. Hernández-Ramírez, "Dualbeam focused ion beam (FIB): a prototyping tool for micro and nanofabrication," Microelectronic Engineering, vol. 84, no. 5-8, pp. 789-792, 2007.

[9] I. Utke, P. Hoffmann, R. Berger, and L. Scandella, "Highresolution magnetic Co supertips grown by a focused electron beam," Applied Physics Letters, vol. 80, no. 25, pp. 4792-4794, 2002.

[10] A. Fernández-Pacheco, J. M. De Teresa, R. Córdoba, and M. R. Ibarra, "Magnetotransport properties of high-quality cobalt nanowires grown by focused-electron-beam-induced deposition," Journal of Physics D, vol. 42, no. 5, Article ID 055005, 6 pages, 2009.

[11] E. S. Sadki, S. Ooi, and K. Hirata, "Focused-ion-beam-induced deposition of superconducting nanowires," Applied Physics Letters, vol. 85, no. 25, pp. 6206-6208, 2004.

[12] D. Spoddig, K. Schindler, P. Rödiger, et al., "Transport properties and growth parameters of $\mathrm{PdC}$ and WC nanowires prepared in a dual-beam microscope," Nanotechnology, vol. 18, no. 49, Article ID 495202, 12 pages, 2007.

[13] I. Guillamón, H. Suderow, S. Vieira, et al., "Nanoscale superconducting properties of amorphous W-based deposits grown with a focused-ion-beam," New Journal of Physics, vol. 10, no. 9, Article ID 093005, 10 pages, 2008.

[14] N. Silvis-Cividjian, C. W. Hagen, and P. Kruit, "Spatial resolution limits in electron-beam-induced deposition," Journal of Applied Physics, vol. 98, no. 8, Article ID 084905, 12 pages, 2005. 
[15] I. Utke, P. Hoffmann, and J. Melngailis, "Gas-assisted focused electron beam and ion beam processing and fabrication," Journal of Vacuum Science and Technology B, vol. 26, no. 4, pp. 1197-1276, 2008.

[16] W. F. van Dorp and C. W. Hagen, "A critical literature review of focused electron beam induced deposition," Journal of Applied Physics, vol. 104, no. 8, Article ID 081301, 42 pages, 2008.

[17] R. M. Langford, T.-X. Wang, and D. Ozkaya, "Reducing the resistivity of electron and ion beam assisted deposited Pt," Microelectronic Engineering, vol. 84, no. 5-8, pp. 784-788, 2007.

[18] J.-F. Lin, J. P. Bird, L. Rotkina, A. Sergeev, and V. Mitin, "Large effects due to electron-phonon-impurity interference in the resistivity of Pt/C-Ga composite nanowires," Applied Physics Letters, vol. 84, no. 19, pp. 3828-3830, 2004.

[19] L. Rotkina, J.-F. Lin, and J. P. Bird, "Nonlinear current-voltage characteristics of Pt nanowires and nanowire transistors fabricated by electron-beam deposition," Applied Physics Letters, vol. 83, no. 21, pp. 4426-4428, 2003.

[20] J.-F. Lin, J. P. Bird, L. Rotkina, and P. A. Bennett, "Classical and quantum transport in focused-ion-beam-deposited $\mathrm{Pt}$ nanointerconnects," Applied Physics Letters, vol. 82, no. 5, pp. 802-804, 2003.

[21] L. Peñate-Quesada, J. Mitra, and P. Dawson, "Non-linear electronic transport in Pt nanowires deposited by focused ion beam," Nanotechnology, vol. 18, no. 21, Article ID 215203, 5 pages, 2007.

[22] A. Fernández-Pacheco, J. M. De Teresa, R. Córdoba, and M. R. Ibarra, "Exploring the conduction in atomic-sized metallic constrictions created by controlled ion etching," Nanotechnology, vol. 19, no. 41, Article ID 415302, 7 pages, 2008.

[23] S. Lipp, L. Frey, C. Lehrer, E. Demm, S. Pauthner, and H. Ryssel, "A comparison of focused ion beam and electron beam induced deposition processes," Microelectronics and Reliability, vol. 36, no. 11-12, pp. 1779-1782, 1996.

[24] M. Takai, W. Jarupoonphol, C. Ochiai, O. Yavas, and Y. K. Park, "Processing of vacuum microelectronic devices by focused ion and electron beams," Applied Physics A, vol. 76, no. 7, pp. 1007-1012, 2003.

[25] M. Prestigiacomo, L. Roussel, A. Houël, et al., "Studies of structures elaborated by focused ion beam induced deposition," Microelectronic Engineering, vol. 76, no. 1-4, pp. 175181,2004

[26] N. Van Lien and B. I. Shklovskii, "Hopping conduction in strong electric fields and directed percolation," Solid State Communications, vol. 38, no. 2, pp. 99-102, 1981.

[27] A. V. Nenashev, F. Jansson, S. D. Baranovskii, R. Österbacka, A. V. Dvurechenskii, and F. Gebhard, "Hopping conduction in strong electric fields: negative differential conductivity," Physical Review B, vol. 78, no. 16, Article ID 165207, 11 pages, 2008.

[28] D. I. Aladashvili, Z. A. Adamiya, K. G. Lavdovskii, E. I. Levin, and B. I. Shklovskii, "Negative differential resistance in the hopping conductivity region in silicon," JETP Letters, vol. 47, no. 8, pp. 466-469, 1988.

[29] A. P. Mel'nikov, Yu. A. Gurvich, L. N. Shestakov, and E. M. Gershenzon, "Magnetic field effects on the nonohmic impurity conduction of uncompensated crystalline silicon," JETP Letters, vol. 73, no. 1, pp. 44-47, 2001.

[30] A. Möbius, C. Frenzel, R. Thielsch, et al., "Metal-insulator transition in amorphous $\mathrm{Si}_{1-x} \mathrm{Ni}_{x}$ : evidence for Mott's minimum metallic conductivity," Physical Review B, vol. 60, no. 20, pp. 14209-14223, 1999.
[31] N. F. Mott and E. A. Davis, Electronic Processes in NonCrystalline Materials, Clarendon Press, Oxford, UK, 1979.

[32] A. L. Efros and B. I. Shklovskii, "Coulomb gap and low temperature conductivity of disordered systems," Journal of Physics C, vol. 8, no. 4, pp. L49-L51, 1975.

[33] Y. Tsukatani, N. Yamasaki, K. Murakami, F. Wakaya, and M. Takai, "Transport properties of Pt nanowires fabricated by beam-induced deposition," Japanese Journal of Applied Physics, vol. 44, no. 7B, pp. 5683-5686, 2005.

[34] Z.-Q. Liu, K. Mitsuishi, and K. Furuya, "Fabrication and investigation of tungsten deposit on top and bottom surfaces of thin film substrate," Japanese Journal of Applied Physics, vol. 46, no. 9B, pp. 6254-6257, 2007.

[35] V. Ambegaokar, B. I. Halperin, and J. S. Langer, "Hopping conductivity in disordered systems," Physical Review B, vol. 4, no. 8, pp. 2612-2620, 1971.

[36] C. Arena, B. Kleinsorge, J. Robertson, W. I. Milne, and M. E. Welland, "Electronic properties of tetrahedral amorphous carbon investigated by scanning tunneling microscopy," Journal of Applied Physics, vol. 85, no. 3, pp. 1609-1615, 1999.

[37] V. Prasad, "Magnetotransport in the amorphous carbon films near the metal-insulator transition," Solid State Communications, vol. 145, no. 4, pp. 186-191, 2008.

[38] O. Massenet, H. Daver, and J. Geneste, "Magnetic ordering and magnetization in amorphous Fe-Ge films," Journal de Physique Colloques, vol. 35, no. C4, pp. 279-285, 1974.

[39] L. Kumari, S. V. Subramanyam, S. Eto, K. Takai, and T. Enoki, "Metal-insulator transition in iodinated amorphous conducting carbon films," Carbon, vol. 42, no. 11, pp. 21332137, 2004.

[40] D. Yu, C. Wang, B. L. Wehrenberg, and P. Guyot-Sionnest, "Variable range hopping conduction in semiconductor nanocrystal solids," Physical Review Letters, vol. 92, no. 21, Article ID 216802, 4 pages, 2004. 

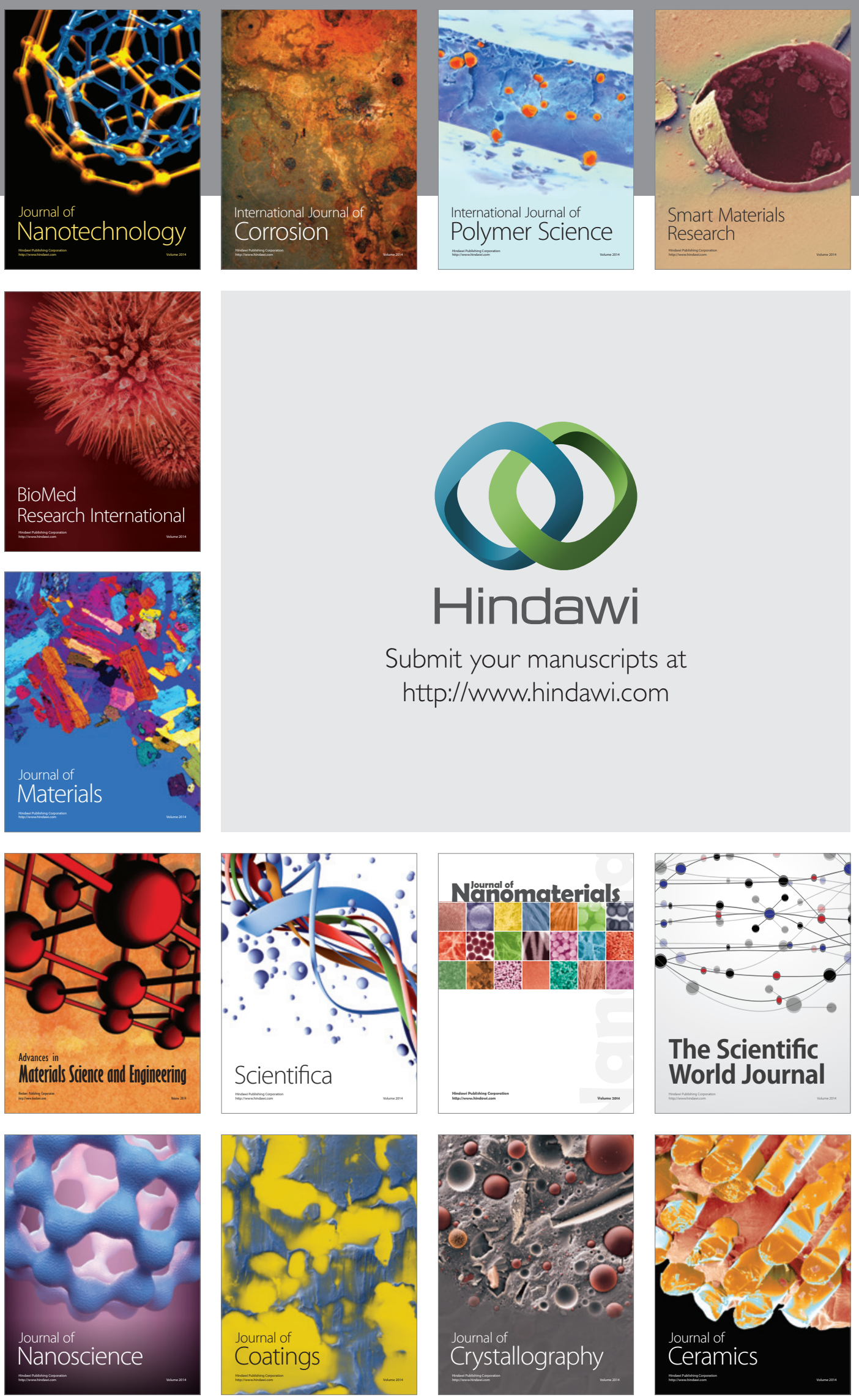

The Scientific World Journal

Submit your manuscripts at

http://www.hindawi.com

\section{World Journal}

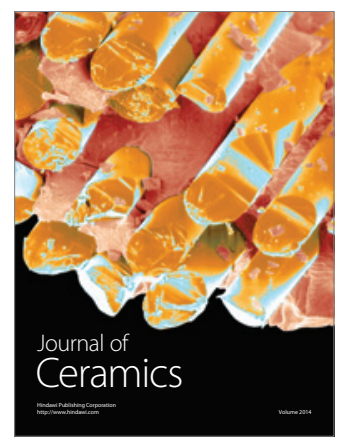

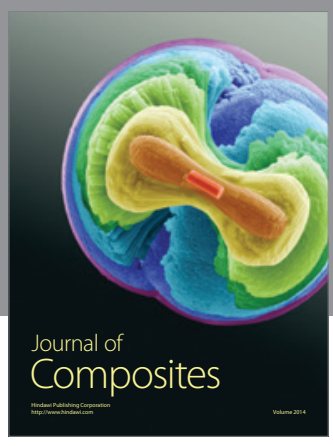
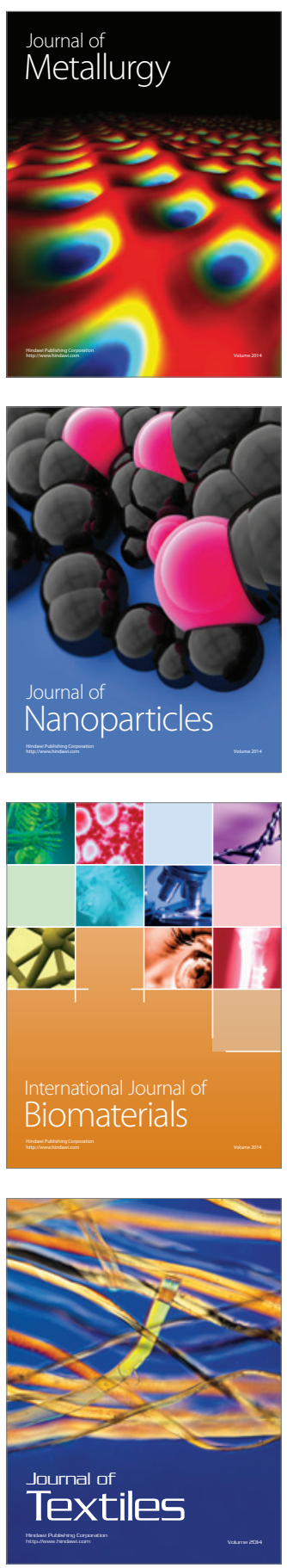